\title{
Myocardial iron overload in thalassemia major. How early to check?
}

\author{
Antonella Meloni ${ }^{*}$, Caterina Borgna-Pignatti ${ }^{2}$, Giulia Guerrini ${ }^{2}$, Vincenzo Positano ${ }^{1}$, Aldo Filosa ${ }^{3}$, \\ Giovan Battista Ruffo ${ }^{4}$, Tommaso Casini ${ }^{5}$, Elisabetta Chiodi ${ }^{6}$, Massimo Lombardi ${ }^{1}$, Alessia Pepe ${ }^{1}$ \\ From 17th Annual SCMR Scientific Sessions \\ New Orleans, LA, USA. 16-19 January 2014
}

\section{Background}

It is still controversy in thalassemia major (TM) if Cardiovascular Magnetic Resonance (CMR) T2* screening should be initiated before the 10 years. To answer this question, we studied retrospectively the prevalence of cardiac iron and function and myocardial fibrosis by CMR in a consistent cohort of TM patients younger than 10 years.

\section{Methods}

From the 2171 patients enrolled in the MIOT (Myocardial Iron Overload in Thalassemia) network, we retrospectively selected the $35 \mathrm{TM}$ patients aged less than 10 years who had undergone at least one MRI scan. Myocardial iron overload (MIO) was measured by $\mathrm{T} 2 *$ multislice multiecho technique. Biventricular function parameters were quantitatively evaluated in a standard way by cine images. To detect myocardial fibrosis, late gadolinium enhancement images were acquired.

\section{Results}

Patients' age ranged from 4.2 to 9.7 years. All MRI scans were performed without sedation. Nine patients $(25.7 \%)$ showed no myocardial iron overload (MIO), 22 patients (62.9\%) showed an heterogeneous MIO with a T2* global value $\geq 20 \mathrm{~ms} ; 2$ patients (5.7\%) showed an heterogeneous MIO and a T2* global value $<20 \mathrm{~ms}$ and 2 patients $(5.7 \%)$ had a homogeneous MIO (Figure 1).
Biventricular function parameters were assessed only in 28/35 patients (80\%), because for 7 patients a short MRI protocol was chosen to avoid sedation. LV dysfunction $(\mathrm{EF}<54 \%)$ was found in one patient (male, 7-year old, treated with deferoxamine and showing an heterogeneous myocardial iron overload with a global T2* value $=$ $31.1 \mathrm{~ms})$. No patient showed RV dysfunction. Finally, 14 patients completed the MRI protocol with acquisition of the LGE images and none of them showed myocardial fibrosis. Table 1 reports the data of the 4 patients ( 3 males and 1 female) with significant myocardial iron overload (global heart T2* $<20 \mathrm{~ms}$ ). The youngest patient was 6 years old, all patient showed no heart dysfunction and in all the iron transfused was less than $35 \mathrm{~g}$.

\section{Conclusions}

The first cardiac $\mathrm{T}^{*}$ assessment should be performed as early as possible without sedation and it is mandatory whenever poor compliance is suspected or if chelation has been started late.

\section{Funding}

The MIOT project receives "no-profit support" from industrial sponsorships (Chiesi Farmaceutici S.p.A. and ApoPharma Inc.). This study was also supported by: "Ministero della Salute, fondi ex art. 12 D.Lgs. 502/92 e s.m.i., ricerca sanitaria finalizzata anno 2006" and "Fondazione L. Giambrone". 


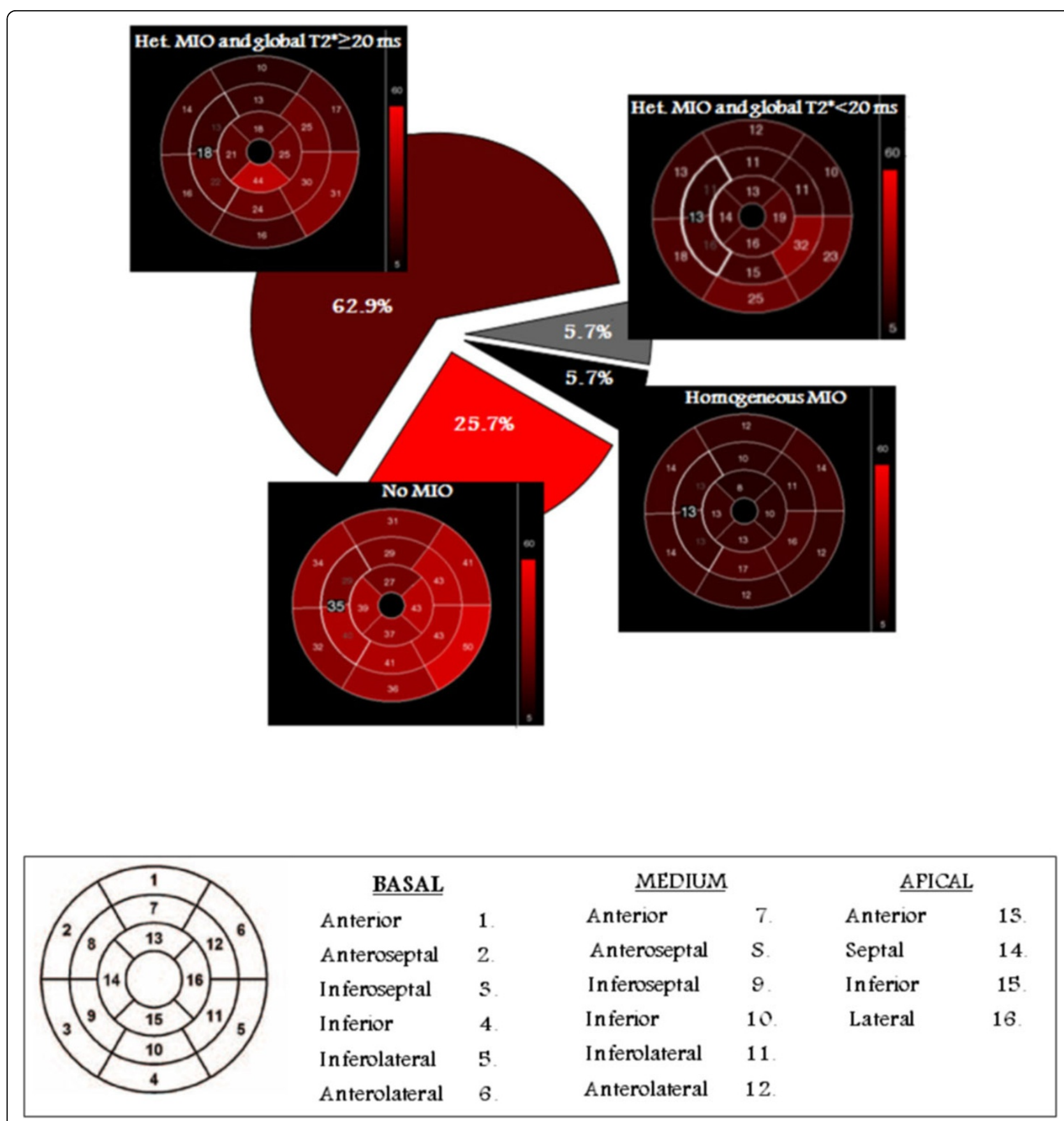

Figure 1 Up: Representative bull's eye maps identifying the 4 patterns of myocardial iron overload (MIO). The pie chart specifies the percentage of patients for each pattern. Bottom: Bull's-eye representation of the 16 myocardial standard segments.

Table 1 Demographic, clinical and MRI data of the 4 patients with global heart T2* $<20$ ms.

\begin{tabular}{|c|c|c|c|c|}
\hline Parameter & Patient 1 & Patient 2 & Patient 3 & Patient 4 \\
\hline Age (yrs) & 9.5 & 6.8 & 8.8 & 7.9 \\
\hline Sex & M & $\mathrm{F}$ & M & M \\
\hline Transfusions starting age (months) & 12 & 7 & 12 & 12 \\
\hline Mean $\mathrm{Hb}$ pre-transfusion $(\mathrm{g} / \mathrm{dl})$ & 9.0 & 9.7 & 9.8 & 9.6 \\
\hline Mean serum ferritin in the previous year $(\mathrm{ng} / \mathrm{ml})$ & 4500 & 2488 & 2579 & 2359 \\
\hline
\end{tabular}


Table 1 Demographic, clinical and MRI data of the 4 patients with global heart T2* ?<? 20 ms. (Continued)

\begin{tabular}{|c|c|c|c|c|}
\hline Transfused iron (g) & 32 & 14 & 23 & 27 \\
\hline Chelation starting age (months) & 16 & 30 & 24 & 36 \\
\hline Chelation treatment at the time of MRI & Deferoxamine & Deferasirox & Deferasirox & Deferoxamine \\
\hline Compliance & good & dubious & excellent & excellent \\
\hline Previous chelation therapy & None & Deferoxamine & Deferoxamine & $\begin{array}{l}\text {-Deferoxamine } \\
\text {-Deferasirox }\end{array}$ \\
\hline Global heart T2*/Mid ventricular septum T2* (ms) & $11.2 / 15$ & $13 / 13$ & $16.2 / 18$ & $18.9 / 24.5$ \\
\hline MRI CIC (mg/g dry weight) & 2.35 & 1.97 & 1.51 & 1.25 \\
\hline N. of pathological segments & 16 & 16 & 12 & 9 \\
\hline Pattern of $\mathrm{MIO}$ & Homogenous & Homogenous & Heterogeneous & Heterogeneous \\
\hline MRI LIC (mg/g dry weight) & 21.4 & 23.3 & 9.6 & 15.1 \\
\hline LV EF (\%) & 61 & $\mathrm{NE}$ & 63 & 59 \\
\hline RV EF (\%) & 63 & $\mathrm{NE}$ & 64 & 56 \\
\hline
\end{tabular}

$\mathrm{M}=$ male, $\mathrm{F}$ = female; $\mathrm{MRI}=$ Magnetic Resonance Imaging; $\mathrm{ClC}=$ cardiac iron concentration; $\mathrm{MIO}=$ myocardial iron overload; $\mathrm{LIC}=$ liver iron concentration; $\mathrm{LV}=$ left ventricular; $\mathrm{EF}=$ ejection fraction; $\mathrm{RV}=$ right ventricular; $\mathrm{NE}=$ not evaluated.

\section{Authors' details}

${ }^{1}$ CMR Unit, Fondazione G.Monasterio CNR-Regione Toscana and Institute of Clinical Physiology, Pisa, Italy. ${ }^{2}$ Department of Clinical and Experimental Medicine (Pediatrics), University of Ferrara, Ferrara, Italy. ${ }^{3}$ UOSD Centro per le Microcitemie, AORN Cardarelli, Napoli, Italy. ${ }^{4}$ U.O.C. Ematologia con Talassemia ARNAS, Ospedale Civico, Palermo, Italy. ${ }^{5}$ Centro Talassemie ed Emoglobinopatie, Ospedale Meyer, Firenze, Italy. ${ }^{6}$ Servizio Radiologia Ospedaliera-Universitaria, Arcispedale "S. Anna" di Ferrara, Ferrara, Italy.

Published: 16 January 2014

doi:10.1186/1532-429X-16-S1-P254

Cite this article as: Meloni et al: Myocardial iron overload in

thalassemia major. How early to check? Journal of Cardiovascular

Magnetic Resonance 2014 16(Suppl 1):P254.

\section{Submit your next manuscript to BioMed Central} and take full advantage of:

- Convenient online submission

- Thorough peer review

- No space constraints or color figure charges

- Immediate publication on acceptance

- Inclusion in PubMed, CAS, Scopus and Google Scholar

- Research which is freely available for redistribution

Submit your manuscript at www.biomedcentral.com/submit 